thyroid gland is indicated inter alia by an accumulation of colloid in the thyroid vesicles. Now in coldblooded animals exposure to cold produces a fall both in the temperature of the tissues of the animal and in the metabolism, while heat raises both. One might expect that a fall in the temperature of the animal as a whole would diminish the activity of its organs, including the thyroid gland. In that case the interesting conclusion would follow that in the course of evolution the response to an environmental stimulus in a specific group of cells has been completely reversed although the cells have not changed their specific character.

Prof. Huxley's suggestion is that the thyroid of coldblooded animals, like that of warm-blooded animals, is stimulated by cold and inhibited by heat. This would imply that while the temperature of the animal as a whole falls and the activity of its organs diminishes, one particular organ - the thyroid glandhas a greater functional activity at a lower temperature than at a higher temperature. One cannot exclude a priori such a possibility because it appears to be paradoxical. But it requires more convincing evidence for its support than Prof. Huxley adduces from his own experiments. The statement attributed to Adler that in tadpoles low temperature caused hypertrophy of the thyroid gland both in growth and functional activity is open to the criticism that in warm-blooded animals increased functional activity does not manifest itself by hypertrophy.

In conclusion, it may be pointed out that the whole problem is further complicated by the fact that in warm-blooded animals the adrenal gland plays a very important part in the heat-regulating mechanism, this gland acting synergically with the thyroid gland. There is a striking parallelism between the develop. ment of the heat-regulating mechanism and the evolution of the adrenal gland as expressed in the changing anatomical relationship of the two histogenetically distinct tissues which in the mammals form cortex and medulla. This must be taken into account when differences in the heat-regulating mechanism of cold-blooded animals is being discussed.

Imperial Cancer Research Fund,

8-11 Queen Square, London, W.C.1, May 13.

\section{Variation of Latitude with the Moon's Position.}

IN NAture of Jan. 26, 1929, p. 127, Prof. H. T. Stetson has described a variation of latitude with the moon's position, and in the Comptes rendus de l'Académie des Sciences of July. 30, 1928, A. Gougenheim has described a variation of latitude with the

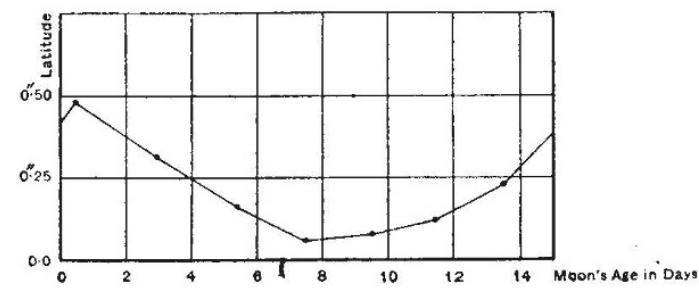

FIG. 1.

age of the moon. In October and November $1926 \mathrm{a}$ series of observations of the latitude of Dehra Dun (India, Lat. $30^{\circ}$ N.) were made with a prismatic astrolabe, which show a clear relation between the latitude and the age of the moon (Fig. 1), but no relation at all between latitude and moon's altitude
(Fig. 2). The variation with the moon's age was about one-third of that found at Algiers, and was apparently in phase with it.

The fact that the astrolabe at Dehra Dun shows no variation with moon's altitude does not of course

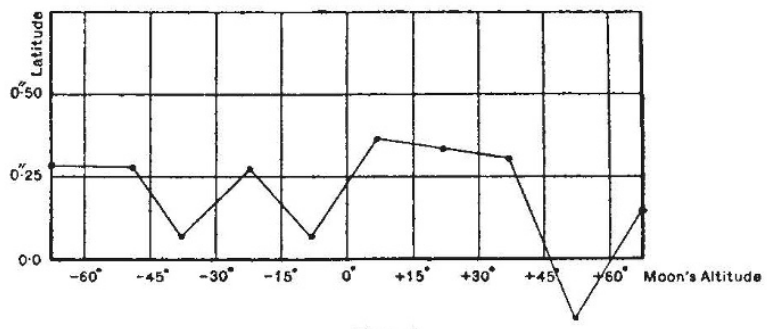

FIG. 2.

invalidate deductions made from Prof. Stetson's more precise and extended series, but it seems surprising that the fortnightly variation (if it truly exists) should be larger and more easily measured than the daily variation.

Each point in Fig. 1 represents about 10 series of observations, each lasting about two hours and giving apparent probable errors of $0^{\prime \prime} \cdot 3$.

Survey of India, Dehra Dun.

\section{G. BomFord.}

\section{A New Titanium Band System.}

The dominant feature of the $M$-type stars is the very extensive group of titanium oxide bands, beginning in the blue region of the spectrum and continuing far to the red. The group of bands occurring in the blue-green region has been analysed (see Christy and Birge, NaturE, 122, $205 ; 1928$ ), and shown to be due to a ${ }^{3} P-{ }^{3} P$ transition of neutral titanium oxide.

Bands in the red portion have been observed by many investigators and especially by P. W. Merrill, who found that those of the $\lambda 7054-\lambda 7700$ region are particularly intense in $M$-type spectra. Twenty bands, including in all 46 heads, extending from $\lambda 7990$ to $\lambda 6270$, and partially overlapping the above group, have now been assigned to a new system. The lower level is the same as that of the former system in the blue-green. This, and the fact that both systems appear in absorption in stellar spectra, show that the two are resonance systems. The frequencies of the heads of the new system are given by $14172 \cdot 2$

$14105 \cdot 8\}+\left(862 \cdot 5 n^{\prime}-3 \cdot 84 n^{\prime 2}\right)-\left(1003 \cdot 8 n^{\prime \prime}-4 \cdot 61 n^{\prime \prime 2}\right)$ $14030 \cdot 8$

with an average residual of $1 \mathrm{~cm}^{-1}$. As shown by the formula, the mean separation of the heads is $70.7 \mathrm{~cm} .^{-1}$. The mean separation of the lower levels of the blue-green system was shown indirectly to be about $70 \mathrm{~cm}^{-1}$ (see Phys. Rev., May 1929), indicating that the upper level of the new system is single. Each of the three heads of the more intense bands has a clearly marked secondary head at about $10 \mathrm{~cm}^{-1}$ to the red. These latter heads are formed presumably by the $Q$ branches. Since transitions between singlets and triplets are very uncommon in band spectra, the upper level is in all probability a ${ }^{3} S$.

The values of $\omega_{0}^{\prime \prime}$ and $\omega_{0}^{\prime \prime} x^{\prime \prime}$ (that is, $1003 \cdot 8$ and $4 \cdot 61$ ) are believed to be somewhat more accurate than those published previously, and are based on the mean separation of levels as found from both systems. Using the new values and assuming a linear extrapolation, the heat of dissociation for the lower level is found to be 6.74 volts. The total energy resulting 